\title{
Parity-Violating Deep Inelastic Scattering
}

\author{
Krishna S. Kumar ${ }^{1 *}$ \\ 1- University of Massachusetts, Amherst - Dept of Physics \\ Amherst, MA 01003 - USA
}

\begin{abstract}
We discuss the physics motivation for an experimental program of parity-violating (PV) asymmetry measurements in the deep inelastic scattering (DIS) of longitudinally polarized electrons off unpolarized fixed targets, made feasible by the intense and stable electron beam at Jefferson Laboratory especially after the planned $12 \mathrm{GeV}$ upgrade. This will provide access to long standing issues in DIS physics, such as a sensitive search for charge symmetry violation at the partonic level, a clean measurement of $d(x) / u(x)$ as $x \rightarrow 1$ and a systematic study of higher-twist effects. Most importantly, it will allow a unique probe of physics at the multi-TeV scale via a precision measurement of the weak neutral current axial-vector quark coupling.
\end{abstract}

\section{Introduction}

Nearly 50 years ago, soon after the discovery of parity violation in beta decay, Zel'dovich speculated that there might be an analogous parity violating neutral current interaction[2]. He noted that if such an interaction existed, then parity violation would be manifested in lepton-nucleon scattering due to the interference between the weak and electromagnetic amplitudes. If one scatters longitudinally polarized electrons off unpolarized protons and flipped the sign of the beam polarization, the fractional difference in the cross-section would be proportional to the 4-momentum transfer $Q^{2} \equiv-\left(p-p^{\prime}\right)^{2}$ expressed in $\mathrm{GeV}^{2}$ :

$$
A_{\mathrm{PV}} \equiv \frac{\sigma_{R}-\sigma_{L}}{\sigma_{R}+\sigma_{L}} \simeq \frac{\left|A_{Z}\right|}{\left|A_{\gamma}\right|} \simeq \frac{G_{F} Q^{2}}{4 \pi \alpha} \simeq 10^{-4} Q^{2}
$$

For typical fixed target experiments, $A_{\mathrm{PV}}$ ranges from roughly $10^{-4}$ to as small as $10^{-7}$. In the mid-seventies, parity violation in deep inelastic electron nucleon scattering was first observed by the SLAC E122 experiment[3], from which the electron-quark weak neutral current coupling could be extracted. The measurement was an important test of the $\mathrm{SU}(2) \times \mathrm{U}(1)$ gauge theory of electroweak interactions, and the extracted value of the electroweak mixing angle $\sin ^{2} \theta_{W}$ matched the corresponding value obtained from neutral current neutrino scattering experiments.

Over the past 20 years, the experimental techniques employed to measure these tiny leftright asymmetries have been steadily refined such that statistical errors approaching 0.01 parts per million (ppm) and systematic errors of a few parts per billion (ppb) are possible[4]. Depending on the choice of target and kinematic variables, this has facilitated measurements in several important physics topics, such as many-body nuclear physics, nucleon structure and searches for physis beyond the standard model at the TeV scale.

The above mentioned physics topics have been pursued using elastic scattering on a variety of targets, with the 4-momentum transfer $Q^{2} \ll 1 \mathrm{GeV}^{2}$. At the higher $Q^{2}$ required to significantly improve on the E122 PVDIS measurement, neither the luminosity nor the experimental technology was available until recently. In this paper, we outline the formalism

\footnotetext{
* funded by US Department of Energy, Division of Nuclear Physics Grant DE-FG02-88R40415-A018
} 
for the analysis of PVDIS measurements and elaborate on the rich physics program that will be enabled, especially with the $12 \mathrm{GeV}$ upgrade of Jefferson Laboratory (JLab).

\section{PVDIS Phenomenology}

$A_{P V}$ in DIS can be written as

$$
\begin{aligned}
A_{P V}= & Q^{2} \frac{G_{F}}{2 \sqrt{2} \pi \alpha}\left[a(x)+\frac{1-(1-y)^{2}}{1+(1-y)^{2}} b(x)\right], \\
& a(x) \equiv \Sigma_{i} f_{i}(x) C_{1 i} q_{i} / \Sigma_{i} f_{i}(x) q_{i}^{2}, \\
& b(x) \equiv \Sigma_{i} f_{i}(x) C_{2 i} q_{i} / \Sigma_{i} f_{i}(x) q_{i}^{2} .
\end{aligned}
$$

Here, $C_{1 i}\left(C_{2 i}\right)$ are the weak vector(axial-vector) weak charges for the $i$ th quark flavor, $x$ is the fraction of the nucleon momentum carried by the struck quark, $f_{i}(x)$ are parton distribution functions and $q_{i}$ are the electromagnetic charges. The $a(x)$ term arises from the product of the electron axial-vector coupling and the quark vector coupling and is typically the dominant term. For an isoscalar target such as deuterium, the dependence on structure largely cancels out in the $A_{P V}$ ratio of the weak and electromagnetic amplitudes:

$$
\begin{gathered}
a(x)=\frac{6}{5}\left[\left(C_{1 u}-\frac{1}{2} C_{1 d}\right)+\text { corrections }\right] ; \\
b(x)=\frac{6}{5}\left[\left(C_{2 u}-\frac{1}{2} C_{2 d}\right) \frac{q(x)-\bar{q}(x)}{q(x)+\bar{q}(x)}+\text { corrections }\right],
\end{gathered}
$$

where $q(x)=u(x)+d(x)$. For scattering off the proton,

$$
a(x)=\left[\frac{u(x)+0.91 d(x)}{u(x)+0.25 d(x)}\right] .
$$

In trying to make precision measurements of $A_{\mathrm{PV}}$ in PVDIS, several important issues should be taken under consideration. The $a(x)$ term is a factor of 5 to 10 larger than the $b(x)$ term because the latter involves the vector coupling of the electron, which is small in the electroweak theory. In trying to test the electroweak theory, it is important to be able to extract the value of the electroweak mixing angle $\sin ^{2} \theta_{W}$ with a relative accuracy better than $1 \%$ in order to be relevant in constraining new physics at the $\mathrm{TeV}$ scale. Consequently, $A_{\mathrm{PV}}$ must be measured to $1 \%$ relative accuracy or better. In order to avoid uncertainties from sea-quark distributions and higher-twist effects, it is important to have $Q^{2} \geq 2 \mathrm{GeV}^{2}$ and $x \geq 0.35$. The DIS cross-section is steeply falling as $x$ increases. The above factors have precluded a followup precision measurement to E122 for the past two decades.

As mentioned in the introduction, the experimental technology in $A_{\mathrm{PV}}$ has now progressed to a point where online background rejection is possible with event rates exceeding $10 \mathrm{MHz}$ and normalization control at the level of $1 \%$ has been achieved [5]. Combined with the success of high current operation at Jefferson Laboratory with a beam energy approaching $6 \mathrm{GeV}$, precision PVDIS measurements become feasible for the first time. Indeed, when the beam energy is upgraded to $11 \mathrm{GeV}$ in the existing experimental halls as planned, high statistics can be accumulated at high $x \sim 0.7$. As we discuss in the following, PVDIS provides access to novel aspects of nucleon structure, complementing and enhancing precision electromagnetic DIS studies. 


\subsection{Charge Symmetry Violation}

As can be seen from Eqn. $5, a(x) \approx 1.15$ for an isoscalar target, independent of $x$. This results from the assumption of charge symmetry, where the $u$-quark distribution in the proton is the same as the $d$-quark distribution in the neutron, with a similar assumption for the proton $d$-quark distribution: $u^{p}=d^{n}$ and $d^{p}=u^{n}$. If $a(x)$ can be measured with high precision over a range of $x$ values, one is thus quite sensitive to charge symmetry violation (CSV). If one defines CSV parameters:

$$
\delta u(x)=u^{p}(x)-d^{n}(x) ; \quad \delta d(x)=d^{p}(x)-u^{n}(x),
$$

then the dependence on the parity-violating asymmetry for an isoscalar target is:

$$
\frac{\delta A_{P V}}{A_{P V}}=0.28 \frac{\delta u-\delta d}{u+d} \equiv 0.28 R_{C S V} .
$$

While $R_{C S V}$ is known to be less than 0.01 for $x<0.4$ from neutrino DIS measurements [6], a bag model calculation suggests that $R_{C S V} \sim 0.01$ for $x \sim 0.4$ and rising to 0.02 for $x \sim 0.6$. At high $x$, knowledge of $u+d$ is limited. As $x \rightarrow 1$, if $u+d$ falls off more rapidly than $\delta u-\delta d$, then $R_{C S V}$ might rise to 0.1 at $x \sim 0.7$, which would be observable with a $1 \% A_{P V}$ measurement. Further, $R_{C S V}$ is quite unconstrained at large $x$. There is the tantalizing possiblity that $R_{C S V}$ in the moderate and high $x$ region is a factor of 3 bigger than above-mentioned values, which would be large enough to explain the $3 \sigma$ discrepancy in the neutrino-nucleon DIS measurement (NuTeV anomaly)[6].

\section{$2.2 d / u$ at high $x$}

As can be seen from Eqn. 7 for PV DIS off the proton, $a(x)$ is quite sensitive to the ratio $d(x) / u(x)$. The value of $d / u$ as $x \rightarrow 1$ is a very important parameter to pin down in DIS physics. It is required in order to properly constrain fits of parton distribution functions and impacts predictions for QCD processes at high energy colliders.

More importantly, $d / u$ at high $x$ provides new information on important pieces of the nucleon wave function. There is empirical evidence that the minority quark in the nucleon is suppressed at high $x$, an intuitive notion in terms of a hyperfine interaction. While the $\mathrm{SU}(6)$ wave function would predict $d / u \sim 0.5$, simple $\mathrm{SU}(6)$-breaking arguments would predict $d / u \sim 0$. However, a perturbative QCD calculation predicts $d / u=0.2$ as $x \rightarrow 1[7]$.

Currently, the best estimates of $d / u$ comes from ${ }^{2} \mathrm{H}$ DIS structure function data, but uncertainties in the ${ }^{2} \mathrm{H}$ wave-function limits the ability to discriminate between various predictions for $d / u[8]$. There are plans to measure $d / u$ via the ratio of ${ }^{3} \mathrm{H}$ and ${ }^{3} \mathrm{He}$ structure functions and also via measurements of deuteron structure functions with tagged slow recoiling protons. A precise enough measurement of $a(x)$ for the proton at $x \sim 0.7$ would be able to distinguish between competing predictions for $d / u$ as $x \rightarrow 1$. The advantage of $A_{P V}$ measurements over other methods is that there are no nuclear corrections since the PVDIS measurement would be made on a proton target.

\subsection{Weak Mixing Angle Measurement}

The measurement of the PV DIS asymmetry to an accuracy of $0.5 \%$ at $Q^{2} \sim 5 \mathrm{GeV}^{2}$ and $x \sim 0.4$ for an isoscalar target such as deuterium, coupled with precise knowledge the 
parameter $a(x)$ from other experiments, the parameter $b(x)$ can be extracted with high accuracy for the first time. This measurement would be robust only if the comprehensive PV DIS program described above is carried out. Indeed, as can be seen from Eqn. 5, $a(x)$ is independent of $x$ and simply a function of $\sin ^{2} \theta_{W}$ under the assumption of charge symmetry and assuming that higher twist effects are either directly measured or constrained. The quantity $b(x)$ is also virtually free of structure function uncertainties at high $x$.

This measurement would test the WNC amplitude in the lepton-quark sector, where there is currently a $3 \sigma$ discrepancy in the $\mathrm{NuTeV}$ result. Furthermore, combined with other measurements in elastic electron-proton scattering, precise constraints would be possible on the lesser known axial-vector quark couplings $C_{2 i}$. This would, among other things, provide complementary constraints on various models with new heavy $Z^{\prime}$ bosons [9].

\subsection{Experimental Equipment for PV DIS at high $x$}

To comprehensively address the physics topics discussed above experimentally, a series of $A_{P V}$ measurements in the range of 1 to $2 \%$ accuracy are required for the $x$ range from 0.3 to 0.7 , with a lever arm of a factor of 2 in $Q^{2}$ while keeping $W_{\min }^{2}>4$ and $Q_{\min }^{2}>1$. With the upgrade of Jlab, high luminosity with a beam energy of $11 \mathrm{GeV}$ becomes possible for the first time. However, to achieve sufficient statistics at the highest possible $Q^{2}$, a spectrometer with at least $50 \%$ acceptance in the azimuth is required.

A large magnet is required to shield the detectors from target photons and to sweep out low energy charged particles. Recent studies have indicated that a solenoid would be ideal for the required geometry, mainly the long target length and the large laboratory scattering angles. Feasibility studies and detailed simulations studies have just begun, exploring the possibility to use one of the existing collider detector solenoids.

\section{Summary}

Parity-violating electron scattering is a mature field and addresses fundamental questions in a variety of different topics. Future measurements at Jefferson Laboratory will lead to important new insights on the structure of the nucleon and yield new and more precise measurements of the weak mixing angle.

\section{References}

[1] Slides: http://indico. cern. ch/contributionDisplay py? contribId=262\&sessionId=10\&conf Id=9499

[2] Ya.B. Zel'dovich,Ya.B., J.Exptl.Theoret.Phys. (U.S.S.R.), 36964 (1959).

[3] C.Y. Prescott et al., Phys.Lett. B84 524 (1979).

[4] K.S. Kumar and P.A. Souder, Prog.Part.Nucl.Phys. 45, S333 (2000).

[5] F.E. Maas et al., Phys.Rev.Lett. 94152001 (2005); D.S. Armstrong et al., Phys.Rev.Lett. 95092001 (2005); A. Acha et al., Phys.Rev.Lett. 98032301 (2007).

[6] J.T. Londergan and A.W. Thomas, hep-ph/0407247 (2004), and references therein.

[7] G.R. Farrar and R.D. Jackson, Phys.Rev.Lett. 43246 (1979).

[8] W. Melnitchouk et al., Phys.Rev.Lett. 845455 (2000); W. Melnitchouk and A.W. Thomas, Phys.Lett. B377 11 (1996).

[9] M.J. Ramsey-Musolf, Phys.Rev. C60 015501 (1999). 